\title{
A General and Long-Lived Catalyst for the Palladium-Catalyzed Coupling of Aryl Halides with Thiols
}

Manuel A. Fernández-Rodríguez, Qilong Shen and John F. Hartwig

Department of Chemistry, Yale University,

P.O. Box 208107, New Haven, Connecticut 06520-8107

\section{Supporting Information}

Complete list of authors in Reference 16: Alcaraz, M.-L.; Atkinson, S.; Cornwall, P.; Foster, A. C.; Gill, D. M.; Humphries, L. A.; Keegan, P. S.; Kemp, R.; Merifield, E.; Nixon, R. A.; Noble, A. J.; O'Beirne, D.; Patel, Z. M.; Perkins, J.; Rowan, P.; Sadler, P.; Singleton, J. T.; Tornos, J.; Watts, A. J.; Woodland, I. A.

General Methods: All reactions were assembled under inert atmosphere. Reactions were conducted in $4 \mathrm{~mL}$ vials sealed with a cap containing a PTFE septum. All glassware was ovendried, evacuated and purged with nitrogen immediately prior to use. All common reagents were obtained from commercial suppliers and used without further purification. All reaction temperatures refer to bath temperatures. CyPF-t-Bu (1-dicyclohexylphosphino-2-di-tbutylphosphinoethylferroceno) was obtained from Solvias AG and Strem Chemicals and used without purification. Toluene was degassed by purging with nitrogen for 45 min and dried with a solvent purification system containing a $1 \mathrm{~m}$ column of activated alumina. 1,2-Dimethoxyethane (DME, 99.9\% purity, HPLC grade) was used without further purification, but was stored under nitrogen. Reactions performed at $110{ }^{\circ} \mathrm{C}$ in DME (b.p. $=85{ }^{\circ} \mathrm{C}$ ) were conducted using the standard vials and caps cited above; no loss of solvent was observed. ${ }^{1} \mathrm{H}$ and ${ }^{13} \mathrm{C}$ NMR spectra were recorded in $\mathrm{CDCl}_{3}$ on $400 \mathrm{MHz}$ or $500 \mathrm{MHz}$ spectrometers with tetramethylsilane or 
residual protiated solvent used as a reference. Abbreviations for ${ }^{1} \mathrm{H}$ NMR splitting patterns are: $\mathrm{s}$, singlet; bs, broad singlet; d, doublet; t, triplet; q, quartet; quint, quintet; sext, sextet; sept, septet; oct, octet; dd, doublet of doublets; dt, doublet of triplets; td, triplet of doublets; tt, triplet of triplets; m, multiplet. The coupling constants are reported in hertz $(\mathrm{Hz})$. Flash column chromatography was carried out on silica gel (230-240 mesh). The yields of the coupled products included in all tables refer to isolated yields and are the average of two runs. Products that had been reported previously were isolated in greater than $95 \%$ purity, as determined by ${ }^{1} \mathrm{H}$ NMR spectroscopy and capillary gas chromatography (GC). GC analyses were obtained with a DB1301 narrow bore column suitable for use with a fast temperature ramp (max $\left.120{ }^{\circ} \mathrm{C} / \mathrm{min}\right)$. Elemental Analyses were performed by Atlantic Microlab, Inc., Norcross, Georgia 30071.

Preparation of stock solution A $\left(1.0 \times 10^{-2} \mathbf{M}\right)$ : DME $(1.0 \mathrm{~mL})$ was added to a mixture of $\mathrm{Pd}(\mathrm{OAc})_{2}(2.2 \mathrm{mg})$ and CyPF-t-Bu $(5.5 \mathrm{mg})$. The resulting orange solution was stirred at room temperature for $1 \mathrm{~min}$ before using.

Preparation of stock solution $\mathbf{B}\left(1.0 \times \mathbf{1 0}^{-2} \mathbf{M}\right)$ : Toluene $(1.0 \mathrm{~mL})$ was added to a mixture of $\mathrm{Pd}(\mathrm{dba})_{2}(2.2 \mathrm{mg})$ and CyPF- $t$-Bu $(5.5 \mathrm{mg})$. The resulting purple solution was stirred at room temperature for 1 min before using.

Preparation of stock solution $\mathbf{C}\left(1.0 \times 10^{-4} \mathbf{M}\right): 10 \mu \mathrm{L}$ of stock solution $\mathbf{A}$ was diluted to $1.0 \mathrm{~mL}$ with DME. The resulting pale yellow solution was stirred at room temperature for $1 \mathrm{~min}$ before using.

General procedure for the palladium-catalyzed coupling of aryl chlorides with aliphatic thiols: The appropriate quantity of stock solution $\mathbf{A}$ was added to a $4 \mathrm{~mL}$ vial containing the aryl chloride $(1.00 \mathrm{mmol})$ and sodium tert-butoxide $(106 \mathrm{mg}, 1.10 \mathrm{mmol})$ in $1.5 \mathrm{~mL}$ of DME. The aliphatic thiol $(1.00 \mathrm{mmol})$ was then added, and the vial sealed with a cap containing a PTFE 
septum. The mixture was heated at $110^{\circ} \mathrm{C}$ until the chloroarene was consumed, as determined by GC. Silica gel $(0.5 \mathrm{~g})$ was added, and the solvents were evaporated under reduced pressure. The crude residue was purified by column chromatography on silica gel using hexane or a mixture of hexane and ethyl acetate as eluent. Aryl sulfides were isolated in the yields reported in Table 1.

General procedure for the palladium-catalyzed coupling of aryl chlorides with aromatic

thiols: The appropriate quantity of stock solution $\mathbf{B}$ was added to a $4 \mathrm{~mL}$ vial containing the aryl chloride $(1.00 \mathrm{mmol})$ and potasium tert-butoxide $(123 \mathrm{mg}, 1.10 \mathrm{mmol})$ in $1.5 \mathrm{~mL}$ of toluene. The aromatic thiol $(1.00 \mathrm{mmol})$ was then added, and the vial sealed with a cap containing a PTFE septum. The mixture was heated at $110^{\circ} \mathrm{C}$ until the chloroarene was consumed, as determined by GC. Silica gel $(0.5 \mathrm{~g})$ was then added, and solvents were evaporated under reduced pressure. The crude residue was purified by column chromatography on silica gel using hexane or a mixture of hexane and ethyl acetate as eluent. Aryl sulfides were isolated in the yields reported in Table 1.

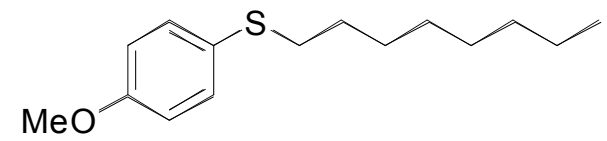

4-Methoxyphenyl octyl sulfide (Table 1, entry 1). ${ }^{1} 100 \mu \mathrm{L}$ of stock solution A were used. 98\% yield. Colorless liquid. ${ }^{1} \mathrm{H}$ NMR $\left(\mathrm{CDCl}_{3}\right): \delta 7.25(\mathrm{~d}, J=8.6 \mathrm{~Hz}, 2 \mathrm{H}), 6.76(\mathrm{~d}, J=8.6 \mathrm{~Hz}$, 2H), $3.71(\mathrm{~s}, 3 \mathrm{H}$ ), 2.73 (t, $J=7.4 \mathrm{~Hz}, 2 \mathrm{H}$ ), 1.50 (quint, $J=7.4 \mathrm{~Hz}, 2 \mathrm{H}$ ), 1.30 (quint, $J=7.4 \mathrm{~Hz}$, 2H), 1.22-1.14 (m, 8H), $0.80(\mathrm{t}, J=7.1 \mathrm{~Hz}, 3 \mathrm{H}) .{ }^{13} \mathrm{C} \mathrm{NMR}\left(\mathrm{CDCl}_{3}\right): \delta 158.6,132.8(2 \mathrm{C}), 126.9$, 114.4 (2C), 55.2, 35.7, 31.7, 29.3, 29.09, 29.06, 28.6, 22.6, 14.0.

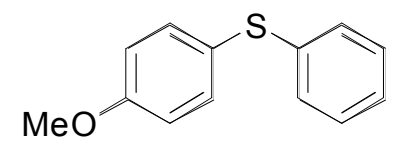

4-Methoxyphenyl phenyl sulfide (Table 1, entry 2). ${ }^{2} 100 \mu \mathrm{L}$ of stock solution $\mathbf{B}$ were used. 98\% yield. Colorless liquid. ${ }^{1} \mathrm{H}$ NMR $\left(\mathrm{CDCl}_{3}\right): \delta 7.23(\mathrm{~d}, J=8.8 \mathrm{~Hz}, 2 \mathrm{H}), 7.06-7.02(\mathrm{~m}, 2 \mathrm{H})$, 
7.00-6.93 (m, 3H), $6.71(\mathrm{~d}, J=8.8 \mathrm{~Hz}, 2 \mathrm{H}), 3.62(\mathrm{~s}, 3 \mathrm{H}) .{ }^{13} \mathrm{C} \mathrm{NMR}\left(\mathrm{CDCl}_{3}\right): \delta 159.7,138.5$, 135.3 (2C), $128.8(2 \mathrm{C}), 128.1(2 \mathrm{C}), 125.7,124.2,114.9(2 \mathrm{C}), 55.3$.

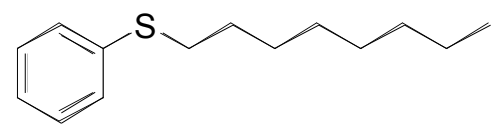

Octyl phenyl sulfide (Table 1, entry 3). ${ }^{3} 50 \mu \mathrm{L}$ of stock solution A were used. 91\% yield. Colorless liquid. ${ }^{1} \mathrm{H}$ NMR $\left(\mathrm{CDCl}_{3}\right)$ : $\delta$ 7.25-7.23 (m, 2H), 7.21-7.16 (m, 2H), 7.09-7.06 (m, 1H), $2.83(\mathrm{t}, J=7.4 \mathrm{~Hz}, 2 \mathrm{H}), 1.57$ (quint, $J=7.4 \mathrm{~Hz}, 2 \mathrm{H}$ ), 1.34 (quint, $J=7.4 \mathrm{~Hz}, 2 \mathrm{H}$ ), 1.21-1.18 (m, $8 \mathrm{H}), 0.80(\mathrm{t}, J=7.1 \mathrm{~Hz}, 3 \mathrm{H}) .{ }^{13} \mathrm{C} \mathrm{NMR}\left(\mathrm{CDCl}_{3}\right): \delta 137.0,128.7$ (4C), 125.5, 33.5, 31.7, 29.09, $29.06(2 \mathrm{C}), 28.8,22.6,14.0$.

Octyl phenyl sulfide (Table 1, entry 4). $10 \mu \mathrm{L}$ of stock solution A were used. 85\% yield.

Preparation of stock solution D (1.0 x 10 $\left.\mathbf{1 0}^{-2} \mathbf{M}\right): \mathrm{Pd}(\mathrm{OAc})_{2}(2.2 \mathrm{mg})$ and CyPF- $t$-Bu $(5.5 \mathrm{mg})$ were added in air to a $4 \mathrm{~mL}$ vial. The flask was sealed with a cap containing a PTFE septum and then evacuated and backfilled with $\mathrm{N}_{2}$. DME $(1.0 \mathrm{~mL})$ was then added to the vial by syringe, and the resulting orange solution was stirred at room temperature for $1 \mathrm{~min}$ before using.

Representative procedure without using a drybox. A. 1 mmol scale: An oven-dried test tube with a screw cap containing a PTFE-lined septum was evacuated and backfilled with $\mathrm{N}_{2}$. To the flask was added $\mathrm{NaO} t \mathrm{Bu}(106 \mathrm{mg}, 1.10 \mathrm{mmol})$ and a stirring bar. The flask was evacuated and heated to remove the moisture present in the base; then evacuated and backfilled with $\mathrm{N}_{2}$ three times. To the flask was then added chlorobenzene (102 $\mu \mathrm{L}, 1.00 \mathrm{mmol})$, DME (2.0 mL), 50 $\mu \mathrm{L}$ of stock solution $\mathbf{D}$, and 1-octanethiol $(173 \mu \mathrm{L}, 1.00 \mathrm{mmol})$, which were stored and handled under an inert atmosphere. The resulting mixture was stirred for $6 \mathrm{~h}$ at $110^{\circ} \mathrm{C}$ until the chlorobenzene was consumed, as determined by GC. Silica gel $(0.5 \mathrm{~g})$ was then added, and solvents were evaporated under reduced pressure. The crude residue was purified by column 
chromatography on silica gel using hexane as eluent to give $205 \mathrm{mg}$ ( $92 \%$ yield) of octyl phenyl sulfide as a colorless liquid.

B. (5 mmol scale): $\mathrm{NaO} t \mathrm{Bu}(0.53 \mathrm{~g}, 5.50 \mathrm{mmol})$, chlorobenzene $(0.51 \mathrm{~mL}, 5.00 \mathrm{mmol})$, $\operatorname{DME}(10.0 \mathrm{~mL}), 250 \mu \mathrm{L}$ of stock solution $\mathbf{D}$, and 1-octanethiol $(0.87 \mathrm{~mL}, 5.00 \mathrm{mmol})$ were used under the same procedure described above to give, after $18 \mathrm{~h}, 1.03 \mathrm{~g}$ (93\% yield) of octyl phenyl sulfide as a colorless liquid.

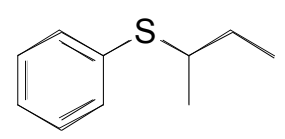

1-Methylpropyl phenyl sulfide (Table 1, entry 5). ${ }^{4} 50 \mu \mathrm{L}$ of stock solution A were used. 91\% yield. Colorless liquid. ${ }^{1} \mathrm{H}$ NMR $\left(\mathrm{CDCl}_{3}\right): \delta$ 7.32-7.29 (m, 2H), 7.20-7.16 (m, 2H), 7.13$7.09(\mathrm{~m}, 1 \mathrm{H}), 3.07$ (sext, $J=6.6 \mathrm{~Hz}, 1 \mathrm{H}), 1.57(\mathrm{~m}, 1 \mathrm{H}), 1.45(\mathrm{~m}, 1 \mathrm{H}), 1.18(\mathrm{~d}, J=6.6 \mathrm{~Hz}, 3 \mathrm{H})$, $0.92(\mathrm{t}, J=7.4 \mathrm{~Hz}, 3 \mathrm{H}) .{ }^{13} \mathrm{C} \mathrm{NMR}\left(\mathrm{CDCl}_{3}\right): \delta 135.4,131.7(2 \mathrm{C}), 128.6(2 \mathrm{C}), 126.4,44.7,29.4$, 20.4, 11.4 .

1-Methylpropyl phenyl sulfide (Table 1, entry 6). A solution of $\mathrm{Pd}(\mathrm{OAc})_{2}(4.4 \mathrm{mg})$ and CyPF-t-Bu $(11 \mathrm{mg})$ in DME $(1 \mathrm{~mL})$ was used as catalyst; the reaction was conducted at $70{ }^{\circ} \mathrm{C}$. $89 \%$ yield.

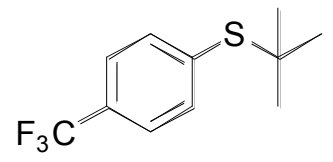

2-Methyl-2-propyl 4-trifluoromethylphenyl sulfide (Table 1, entry 7). $100 \mu \mathrm{L}$ of stock solution A were used. 82\% yield. Colorless liquid. ${ }^{1} \mathrm{H}$ NMR $\left(\mathrm{CDCl}_{3}\right): \delta 7.56(\mathrm{~d}, J=7.9 \mathrm{~Hz}, 2 \mathrm{H})$, $7.50(\mathrm{~d}, J=7.9 \mathrm{~Hz}, 2 \mathrm{H}), 1.23(\mathrm{~s}, 9 \mathrm{H}) .{ }^{13} \mathrm{C} \mathrm{NMR}\left(\mathrm{CDCl}_{3}\right): \delta 137.6,137.3(2 \mathrm{C}), 130.5\left(\mathrm{q},{ }^{2} J_{\mathrm{C}-F}=\right.$ $32.6 \mathrm{~Hz}), 125.2\left(\mathrm{q},{ }^{3} J_{C-F}=3.8 \mathrm{~Hz}\right), 124.5\left(\mathrm{q},{ }^{1} J_{C-F}=272.5 \mathrm{~Hz}\right), 46.6,30.9$ (3C). Elem. Anal. Calcd for $\mathrm{C}_{11} \mathrm{H}_{13} \mathrm{~F}_{3} \mathrm{~S}: \mathrm{C}, 56.39 ; \mathrm{H}, 5.59$. Found: C, 56.59; H, 5.60. 


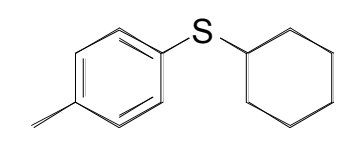

Cyclohexyl 4-methylphenyl sulfide (Table 1, entry 8). ${ }^{5} 50 \mu \mathrm{L}$ of stock solution A were used. $98 \%$ yield. Colorless liquid. ${ }^{1} \mathrm{H}$ NMR $\left(\mathrm{CDCl}_{3}\right): \delta 7.23(\mathrm{~d}, J=8.0 \mathrm{~Hz}, 2 \mathrm{H}), 7.01(\mathrm{~d}, J=8.0$ $\mathrm{Hz}, 2 \mathrm{H}), 2.93(\mathrm{tt}, J=10.5 \mathrm{~Hz}$ and $3.8 \mathrm{~Hz}, 1 \mathrm{H}), 2.24(\mathrm{~s}, 3 \mathrm{H}), 1.89-1.86(\mathrm{~m}, 2 \mathrm{H}), 1.69-1.66(\mathrm{~m}, 2$ $\mathrm{H}), 1.53-1.49(\mathrm{~m}, 1 \mathrm{H}), 1.30-1.11(\mathrm{~m}, 5 \mathrm{H}) .{ }^{13} \mathrm{C} \mathrm{NMR}\left(\mathrm{CDCl}_{3}\right): \delta 136.7,132.7(2 \mathrm{C}), 131.1,129.4$ (2C), 47.0, $33.3(2 \mathrm{C}), 26.0,25.7(2 \mathrm{C}), 21.0$.

Cyclohexyl 4-methylphenyl sulfide (Table 1, entry 9). A solution of $\mathrm{Pd}(\mathrm{OAc})_{2}(4.4 \mathrm{mg})$ and CyPF-t-Bu $(11 \mathrm{mg})$ in DME $(1 \mathrm{~mL})$ was used as catalyst; the reaction was conducted at 70 ${ }^{\circ}$ C. $91 \%$ yield.

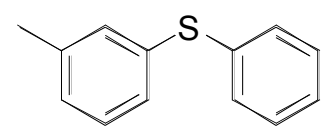

3-Methylphenyl phenyl sulfide (Table 1, entry 10). ${ }^{6} 100 \mu \mathrm{L}$ of stock solution B were used. 98\% yield. Colorless liquid. ${ }^{1} \mathrm{H}$ NMR $\left(\mathrm{CDCl}_{3}\right): \delta$ 7.22-7.20 (m, 2H), 7.17-7.14 (m, 2H), 7.11$7.03(\mathrm{~m}, 4 \mathrm{H}), 6.92(\mathrm{~m}, 1 \mathrm{H}), 2.18(\mathrm{~s}, 3 \mathrm{H}) .{ }^{13} \mathrm{C} \mathrm{NMR}\left(\mathrm{CDCl}_{3}\right): \delta 138.9,136.0,135.1,131.7,130.6$ (2C), 129.0 (2C), 128.9, 128.2, 127.9, 126.7, 21.2.

3-Methylphenyl phenyl sulfide (Table 1, entry 11). $50 \mu \mathrm{L}$ of stock solution B were used. $86 \%$ yield.

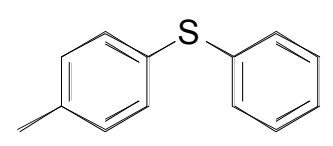

4-Methylphenyl phenyl sulfide (Table 1 , entry 12). ${ }^{2} 100 \mu \mathrm{L}$ of stock solution B were used. 95\% yield. Colorless liquid. ${ }^{1} \mathrm{H}$ NMR $\left(\mathrm{CDCl}_{3}\right): \delta 7.21(\mathrm{~d}, J=8.0 \mathrm{~Hz}, 2 \mathrm{H}), 7.19-7.14(\mathrm{~m}, 4 \mathrm{H})$, 7.11-7.05 (m, 1H), $7.03(\mathrm{~d}, J=8.0 \mathrm{~Hz}, 2 \mathrm{H}), 2.25(\mathrm{~s}, 3 \mathrm{H}) .{ }^{13} \mathrm{C} \mathrm{NMR}\left(\mathrm{CDCl}_{3}\right): \delta 137.5,137.0$, 132.2 (2C), 131.2, $130.0(2 \mathrm{C}), 129.7$ (2C), 128.9 (2C), 126.3, 21.0. 
4-Methylphenyl phenyl sulfide (Table 1, entry 13). A solution of $\operatorname{Pd}(d b a)_{2}(6.6 \mathrm{mg})$ and CyPF-t-Bu $(16.5 \mathrm{mg})$ in toluene $(1.0 \mathrm{~mL})$ was used as catalyst; the reaction was heated at $70{ }^{\circ} \mathrm{C}$ until no further reaction was observed ( $93 \%$ conversion). $83 \%$ yield.

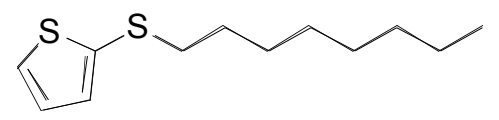

Octyl 2-thiophenyl sulfide (Table 1, entry 14). ${ }^{7} 50 \mu \mathrm{L}$ of stock solution A were used. $96 \%$ yield. Colorless liquid. ${ }^{1} \mathrm{H}$ NMR $\left(\mathrm{CDCl}_{3}\right): \delta 7.23(\mathrm{dd}, J=5.4 \mathrm{~Hz}$ and $1.3 \mathrm{~Hz}, 1 \mathrm{H}), 7.02(\mathrm{dd}, J=$ $3.5 \mathrm{~Hz}$ and $1.3 \mathrm{~Hz}, 1 \mathrm{H}), 6.88(\mathrm{dd}, J=5.4 \mathrm{~Hz}$ and $3.5 \mathrm{~Hz}, 1 \mathrm{H}), 2.70(\mathrm{t}, J=7.3 \mathrm{~Hz}, 2 \mathrm{H}), 1.53$ (quint, $J=7.3 \mathrm{~Hz}, 2 \mathrm{H}), 1.33-1.27(\mathrm{~m}, 2 \mathrm{H}), 1.20(\mathrm{bs}, 8 \mathrm{H}), 0.80(\mathrm{t}, J=6.9 \mathrm{~Hz}, 3 \mathrm{H}) .{ }^{13} \mathrm{C} \mathrm{NMR}$ $\left(\mathrm{CDCl}_{3}\right): \delta 134.9,133.1,128.7,127.3,38.9,31.7,29.3,29.1,29.0,28.3,22.6,14.0$.

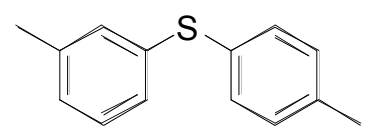

3-Methylphenyl 4-Methylphenyl sulfide (Table 1, entry 15). ${ }^{8} 100 \mu \mathrm{L}$ of stock solution B and LiHMDS (184 mg, $1.10 \mathrm{mmol})$ were used. 93\% yield. Colorless liquid. ${ }^{1} \mathrm{H} \mathrm{NMR}\left(\mathrm{CDCl}_{3}\right)$ : d 7.28-7.25 (m, 2H), 7.16-7.07 (m, 5H), $6.97(\mathrm{~d}, J=7.3 \mathrm{~Hz}, 1 \mathrm{H}), 2.31(\mathrm{~s}, 3 \mathrm{H}), 2.26(\mathrm{~s}, 3 \mathrm{H}) .{ }^{13} \mathrm{C}$ NMR $\left(\mathrm{CDCl}_{3}\right): \delta 138.7,137.2,136.5,131.9(2 \mathrm{C}), 131.5,130.5,129.9$ (2C), 128.8, 127.3, 127.0, 21.2, 21.0.

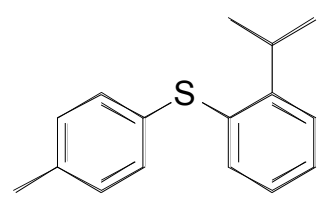

2-Isopropylphenyl 4-methylphenyl sulfide (Table 1, entry 16). $250 \mu \mathrm{L}$ of stock solution B were used. 97\% yield. Colorless liquid. ${ }^{1} \mathrm{H}$ NMR $\left(\mathrm{CDCl}_{3}\right): \delta 7.32(\mathrm{dd}, J=7.7 \mathrm{~Hz}$ and $1.4 \mathrm{~Hz}$, 1H), 7.26-7.14 (m, 4H), 7.11-7.06 (m, 3H), 3.54 (sept, $J=6.8 \mathrm{~Hz}, 1 \mathrm{H}), 2.32(\mathrm{~s}, 3 \mathrm{H}), 1.22(\mathrm{~d}, J=$ $6.8 \mathrm{~Hz}, 6 \mathrm{H}) .{ }^{13} \mathrm{C} \mathrm{NMR}\left(\mathrm{CDCl}_{3}\right): \delta 149.2,136.4,133.8,132.7,132.3,130.6(2 \mathrm{C}), 129.8(2 \mathrm{C})$, 
127.6, 126.4, 125.7, 30.4, 23.4 (2C), 20.9. Elem. Anal. Calcd for $\mathrm{C}_{16} \mathrm{H}_{18} \mathrm{~S}$ : C, 79.29; H, 7.49. Found: C, 78.99; H, 7.57.

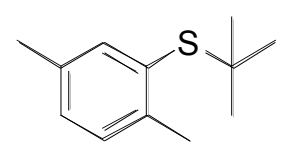

2,5-Dimethylphenyl 2-methyl-2-propyl sulfide (Table 1, entry 17). ${ }^{9} 500 \mu \mathrm{L}$ of stock solution A were used. 89\% yield. Colorless liquid. ${ }^{1} \mathrm{H}$ NMR $\left(\mathrm{CDCl}_{3}\right): \delta 7.26(\mathrm{~s}, 1 \mathrm{H}), 7.06(\mathrm{~d}, J=$ $7.9 \mathrm{~Hz}, 1 \mathrm{H}), 6.96(\mathrm{~d}, J=7.9 \mathrm{~Hz}, 1 \mathrm{H}), 2.38(\mathrm{~s}, 3 \mathrm{H}), 2.21(\mathrm{~s}, 3 \mathrm{H}), 1.20(\mathrm{~s}, 9 \mathrm{H}) .{ }^{13} \mathrm{C} \mathrm{NMR}\left(\mathrm{CDCl}_{3}\right)$ : $\delta$ 140.5, 139.4, 135.0, 131.7, 130.1, 129.6, 46.9, 31.0 (3C), 21.2, 20.6.

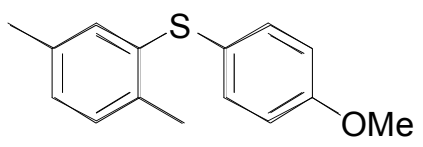

2,5-Dimethylphenyl 4-methoxyphenyl sulfide (Table 1, entry 18). ${ }^{9} 1000 \mu \mathrm{L}$ of stock solution B were used. 92\% yield. Colorless liquid. ${ }^{1} \mathrm{H} \mathrm{NMR}\left(\mathrm{CDCl}_{3}\right): \delta 7.27(\mathrm{~d}, J=8.8 \mathrm{~Hz}, 2 \mathrm{H})$, $7.03(\mathrm{~d}, J=7.6 \mathrm{~Hz}, 1 \mathrm{H}), 6.89(\mathrm{~d}, J=7.6 \mathrm{~Hz}, 1 \mathrm{H}), 6.86-6.82(\mathrm{~m}, 3 \mathrm{H}), 3.75(\mathrm{~s}, 3 \mathrm{H}), 2.31(\mathrm{~s}, 3 \mathrm{H})$, $2.18(\mathrm{~s}, 3 \mathrm{H}) .{ }^{13} \mathrm{C} \mathrm{NMR}\left(\mathrm{CDCl}_{3}\right): \delta 159.2,136.1,135.9,134.3,133.8(2 \mathrm{C}), 130.1,130.0,127.1$, 124.9, 114.8 (2C), 55.1, 20.8, 19.7.

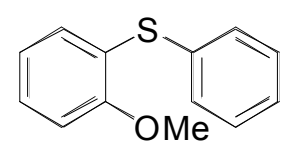

2-Methoxyphenyl phenyl sulfide (Table 1, entry 19). ${ }^{2} 1000 \mu \mathrm{L}$ of stock solution B were used. 97\% yield. Colorless liquid. ${ }^{1} \mathrm{H}$ NMR $\left(\mathrm{CDCl}_{3}\right): \delta$ 7.42-7.40 (m, $\left.2 \mathrm{H}\right), 7.36(\mathrm{t}, J=7.4 \mathrm{~Hz}$, 2H), 7.32-7.27 (m, 2H), 7.14 (dt, $J=7.8 \mathrm{~Hz}$ and $1.7 \mathrm{~Hz}, 1 \mathrm{H}), 6.96-6.90(\mathrm{~m}, 2 \mathrm{H}), 3.91(\mathrm{~s}, 3 \mathrm{H})$. ${ }^{13} \mathrm{C} \mathrm{NMR}\left(\mathrm{CDCl}_{3}\right): \delta 157.1,134.3,131.4,131.3$ (2C), 129.0 (2C), 128.2, 126.9, 123.9, 121.1, 110.7, 55.7 . 
General procedure for the palladium-catalyzed coupling of functionalized aryl chlorides with aliphatic and aromatic thiols.

The appropriate quantity of stock solution $\mathbf{A}$ was added to a $4 \mathrm{~mL}$ vial containing the aryl chloride $(1.00 \mathrm{mmol})$ and $\mathrm{NaO} t \mathrm{Bu}(230 \mathrm{mg}, 2.40 \mathrm{mmol})$ in $1.5 \mathrm{~mL}$ of DME. The thiol (1.00 mmol) was then added, and the vial sealed with a cap containing a PTFE septum. The mixture was heated at $110^{\circ} \mathrm{C}$ until the chloroarene was consumed, as determined by GC. Silica gel $(0.5 \mathrm{~g})$ was added, and the solvents were evaporated under reduced pressure. The crude residue was purified by column chromatography on silica gel using hexane or mixtures of hexane and ethyl acetate as eluent. Aryl sulfides were isolated in the yields reported in Table 1.

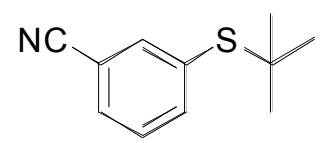

3-Cyanophenyl 2-methyl-2-propyl sulfide (Table 1, entry 20). $50 \mu \mathrm{L}$ of stock solution A and $\mathrm{NaO} t \mathrm{Bu}(106 \mathrm{mg}, 1.10 \mathrm{mmol})$ were used. $90 \%$ yield. White solid. ${ }^{1} \mathrm{H} \mathrm{NMR}\left(\mathrm{CDCl}_{3}\right): \delta 7.82$ (s, 1H), $7.76(\mathrm{~d}, J=7.8 \mathrm{~Hz}, 1 \mathrm{H}), 7.65(\mathrm{~d}, J=7.8 \mathrm{~Hz}, 1 \mathrm{H}), 7.45(\mathrm{t}, J=7.8 \mathrm{~Hz}, 1 \mathrm{H}), 1.30(\mathrm{~s}, 9 \mathrm{H})$. ${ }^{13} \mathrm{C} \mathrm{NMR}\left(\mathrm{CDCl}_{3}\right): \delta 141.5,140.2,134.7,132.0,129.1,118.1,112.6,46.7,30.8$ (3C). Elem. Anal. Calcd for $\mathrm{C}_{11} \mathrm{H}_{13} \mathrm{NS}$ : C, 69.07; H, 6.85; N, 7.32. Found: C, 69.25; H, 6.78; N, 7.25.

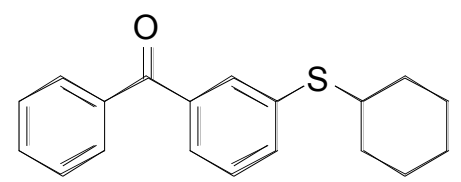

3-Benzoylphenyl cyclohexyl sulfide (Table 1, entry 21). $50 \mu \mathrm{L}$ of stock solution $\mathbf{A}$ and $\mathrm{NaO} t \mathrm{Bu}(106 \mathrm{mg}, 1.10 \mathrm{mmol})$ were used. 86\% yield. Colorless oil. ${ }^{1} \mathrm{H} \mathrm{NMR}\left(\mathrm{CDCl}_{3}\right): \delta 7.90$ $7.78(\mathrm{~m}, 3 \mathrm{H}), 7.62-7.56(\mathrm{~m}, 3 \mathrm{H}), 7.47(\mathrm{t}, J=7.9 \mathrm{~Hz}, 2 \mathrm{H}), 7.38(\mathrm{t}, J=7.8 \mathrm{~Hz}, 1 \mathrm{H}), 3.16(\mathrm{tt}, J=$ $10.4 \mathrm{~Hz}$ and $3.7 \mathrm{~Hz}, 1 \mathrm{H}), 2.0-1.96(\mathrm{~m}, 2 \mathrm{H}), 1.78-1.75(\mathrm{~m}, 2 \mathrm{H}), 1.62-1.59(\mathrm{~m}, 1 \mathrm{H}), 1.42-1.21(\mathrm{~m}$, 5H). ${ }^{13} \mathrm{C} \mathrm{NMR}\left(\mathrm{CDCl}_{3}\right): \delta 195.9,138.0,137.2,136.0,134.9,132.4,132.2,129.9(2 \mathrm{C}), 128.5$, 
128.1 (2C), 127.8, 46.2, 33.0 (2C), 25.8, 25.5 (2C). Elem. Anal. Calcd for $\mathrm{C}_{19} \mathrm{H}_{20} \mathrm{OS}: \mathrm{C}, 76.98 ; \mathrm{H}$, 6.80. Found: C, 76.95; H, 6.79 .

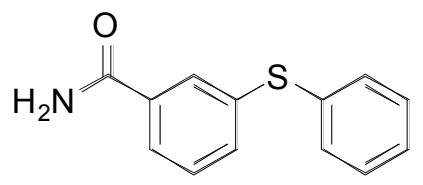

3-Phenylsulfanylbenzamide (Table 1, entry 22) ${ }^{10} 250 \mu \mathrm{L}$ of stock solution A were used. $70 \%$ yield. White solid. ${ }^{1} \mathrm{H}$ NMR $\left(\mathrm{CDCl}_{3}\right): \delta 7.75(\mathrm{t}, J=1.8 \mathrm{~Hz}, 1 \mathrm{H}), 7.65(\mathrm{dt}, J=7.6 \mathrm{~Hz}$ and 1.3 $\mathrm{Hz}, 1 \mathrm{H}), 7.43-7.26(\mathrm{~m}, 7 \mathrm{H}), 6.40-6.18(\mathrm{~m}, 2 \mathrm{H}) .{ }^{13} \mathrm{C} \mathrm{NMR}\left(\mathrm{CDCl}_{3}\right): \delta 168.9,137.4,134.3,134.2$, 133.5, $131.8(2 \mathrm{C}), 129.4(2 \mathrm{C}), 129.3,128.9,127.7,125.6$.

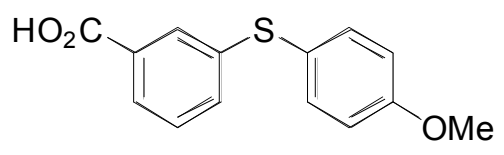

3-(4-Methoxyphenylsulfanyl)benzoic acid (Table 1, entry 23). ${ }^{11} 250 \mu \mathrm{L}$ of stock solution A were used. 74\% yield. White solid. ${ }^{1} \mathrm{H}$ NMR $\left(\mathrm{CDCl}_{3}\right)$ : $\delta 7.90$ (bs, $\left.1 \mathrm{H}\right), 7.86-7.83(\mathrm{~m}, 1 \mathrm{H}), 7.45$ $(\mathrm{d}, J=9.0 \mathrm{~Hz}, 2 \mathrm{H}), 7.36-7.31(\mathrm{~m}, 2 \mathrm{H}), 6.92(\mathrm{~d}, J=9.0 \mathrm{~Hz}, 2 \mathrm{H}), 3.83(\mathrm{~s}, 3 \mathrm{H}) .{ }^{13} \mathrm{C} \mathrm{NMR}\left(\mathrm{CDCl}_{3}\right)$ : $\delta 171.8,160.1,140.1,135.9$ (2C), 132.6, 129.9, 129.0, 128.9, 127.2, 122.8, $115.2(2 \mathrm{C}), 55.3$.

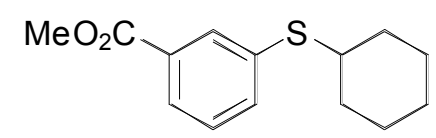

Methyl 3-cyclohexylsulfanylbenzoate (Table 1, entry 24). ${ }^{9} 500 \mu \mathrm{L}$ of stock solution A and $\mathrm{Cs}_{2} \mathrm{CO}_{3}(359 \mathrm{mg}, 1.10 \mathrm{mmol})$ were used. $72 \%$ yield. Pale yellow oil. ${ }^{1} \mathrm{H} \mathrm{NMR}\left(\mathrm{CDCl}_{3}\right): \delta 7.97(\mathrm{~s}$, 1H), $7.78(\mathrm{~d}, J=7.9 \mathrm{~Hz}, 1 \mathrm{H}), 7.48(\mathrm{~d}, J=7.9 \mathrm{~Hz}, 1 \mathrm{H}), 7.28-7.24(\mathrm{~m}, 1 \mathrm{H}), 3.83(\mathrm{~s}, 3 \mathrm{H}), 3.10-$ $3.05(\mathrm{~m}, 1 \mathrm{H}), 1.90-1.86(\mathrm{~m}, 2 \mathrm{H}), 1.69-1.67(\mathrm{~m}, 2 \mathrm{H}), 1.54-1.51(\mathrm{~m}, 1 \mathrm{H}), 1.33-1.15(\mathrm{~m}, 5 \mathrm{H}) .{ }^{13} \mathrm{C}$ NMR $\left(\mathrm{CDCl}_{3}\right): \delta 166.5,136.0,135.8,132.3,130.6,128.6,127.5,52.0,46.4,33.1(2 \mathrm{C}), 25.8$ $25.6(2 \mathrm{C})$ 


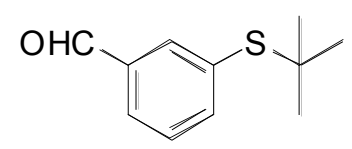

3-(2-Methyl-2-propylsulfanyl)benzaldehyde (Table 1, entry 25). $250 \mu \mathrm{L}$ of stock solution A and $\mathrm{Cs}_{2} \mathrm{CO}_{3}(359 \mathrm{mg}, 1.10 \mathrm{mmol})$ were used. $91 \%$ yield. Colorless oil. ${ }^{1} \mathrm{H}$ NMR $\left(\mathrm{CDCl}_{3}\right)$ : $\delta 10.03(\mathrm{~s}, 1 \mathrm{H}), 8.02(\mathrm{t}, J=1.6 \mathrm{~Hz}, 1 \mathrm{H}), 7.88(\mathrm{dt}, J=7.6 \mathrm{~Hz}$ and $1.6 \mathrm{~Hz}, 1 \mathrm{H}), 7.79(\mathrm{dt}, J=7.6$ $\mathrm{Hz}$ and $1.6 \mathrm{~Hz}, 1 \mathrm{H}), 7.51(\mathrm{t}, J=7.6 \mathrm{~Hz}, 1 \mathrm{H}), 1.31(\mathrm{~s}, 9 \mathrm{H}) .{ }^{13} \mathrm{C} \mathrm{NMR}\left(\mathrm{CDCl}_{3}\right): \delta$ 191.5, 143.0, 138.2, 136.5, 134.2, 129.4, 128.9, 46.2, 30.7 (3C). Elem. Anal. Calcd for $\mathrm{C}_{11} \mathrm{H}_{14} \mathrm{OS}: \mathrm{C}, 68.00 ; \mathrm{H}$, 7.26. Found: C, 67.88; H, 7.45.

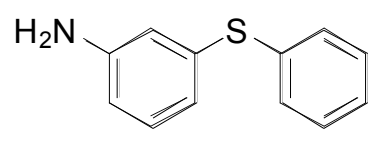

3-Phenylsulfanylaniline (Table 1, entry 26). ${ }^{12} 250 \mu \mathrm{L}$ of stock solution A were used. 91\% yield. Yellow oil. ${ }^{1} \mathrm{H}$ NMR $\left(\mathrm{CDCl}_{3}\right): \delta$ 7.35-7.32 (m, 2H), 7.29-7.20 (m, 3H), $7.05(\mathrm{t}, J=8.1 \mathrm{~Hz}$, $1 \mathrm{H}), 6.73-6.68(\mathrm{~m}, 1 \mathrm{H}), 6.61-6.09(\mathrm{~m}, 1 \mathrm{H}), 6.53-6.50(\mathrm{~m}, 1 \mathrm{H}), 3.60(\mathrm{bs}, 2 \mathrm{H}) .{ }^{13} \mathrm{C} \mathrm{NMR}\left(\mathrm{CDCl}_{3}\right)$ : $\delta$ 147.0, 136.3, 135.6, $130.9(2 \mathrm{C}), 129.8,129.0(2 \mathrm{C}), 126.8,120.8,116.9,113.8$.

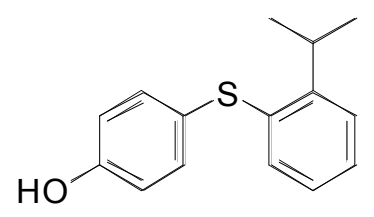

4-(2-Isopropylphenylsulfanyl)phenol (Table 1, entry 27). A solution of $\mathrm{Pd}(\mathrm{OAc})_{2}(4.4 \mathrm{mg})$ and CyPF-t-Bu $(11 \mathrm{mg})$ in DME $(1 \mathrm{~mL})$ was used as catalyst. $91 \%$ yield. Pale yellow oil. ${ }^{1} \mathrm{H}$ $\operatorname{NMR}\left(\mathrm{CDCl}_{3}\right): \delta$ 7.22-7.16 (m, 3H), 7.13-7.08 (m, 1H), 7.00-6.93 (m, 2H), 6.74-6.70 (m, 2H), $5.28(\mathrm{bs}, 1 \mathrm{H}), 3.43(\mathrm{~m}, 1 \mathrm{H}), 1.16(\mathrm{dd}, J=6.8 \mathrm{~Hz}$ and $1.8 \mathrm{~Hz}, 6 \mathrm{H}) \cdot{ }^{13} \mathrm{C} \mathrm{NMR}\left(\mathrm{CDCl}_{3}\right): \delta 155.3$, 147.7, 135.5, 134.4 (2C), 130.1, 126.7, 126.3, 125.6, 125.5, 116.4 (2C), 30.2, 23.2 (2C). Elem. Anal. Calcd for $\mathrm{C}_{15} \mathrm{H}_{16} \mathrm{OS}: \mathrm{C}, 73.73 ; \mathrm{H}, 6.60$. Found: C, 73.45; H, 6.64 . 


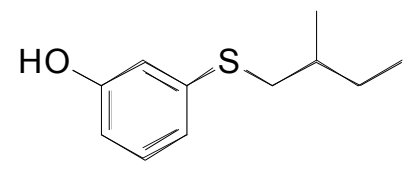

3-(2-Methylbutylsulfanyl)phenol (Table 1, entry 28). $500 \mu \mathrm{L}$ of stock solution A were used. $85 \%$ yield. Yellow oil. ${ }^{1} \mathrm{H}$ NMR $\left(\mathrm{CDCl}_{3}\right): \delta 7.04(\mathrm{t}, J=7.9 \mathrm{~Hz}, 1 \mathrm{H}), 6.80(\mathrm{~m}, 1 \mathrm{H}), 6.72(\mathrm{t}$, $J=2.1 \mathrm{~Hz}, 1 \mathrm{H}), 6.53(\mathrm{~m}, 1 \mathrm{H}), 5.90-4.20(\mathrm{bs}, 1 \mathrm{H}), 2.84(\mathrm{dd}, J=12.3 \mathrm{~Hz}$ and $5.7 \mathrm{~Hz}, 1 \mathrm{H}), 2.65$ $(\mathrm{dd}, J=12.3 \mathrm{~Hz}$ and $7.6 \mathrm{~Hz}, 1 \mathrm{H}), 1.58$ (oct, $J=6.6 \mathrm{~Hz}, 1 \mathrm{H}), 1.49-1.40(\mathrm{~m}, 1 \mathrm{H}), 1.22-1.13(\mathrm{~m}$, 1H), $0.93(\mathrm{~d}, J=6.6 \mathrm{~Hz}, 3 \mathrm{H}), 0.82(\mathrm{~d}, J=7.4 \mathrm{~Hz}, 3 \mathrm{H}) .{ }^{13} \mathrm{C} \mathrm{NMR}\left(\mathrm{CDCl}_{3}\right): \delta 155.6,139.1,129.8$, 120.7, 115.0, 112.5, 40.2, 34.3, 28.7, 18.8, 11.1. Elem. Anal. Calcd for $\mathrm{C}_{11} \mathrm{H}_{16} \mathrm{OS}$ : C, 67.30; $\mathrm{H}$, 8.22. Found: C, 67.52; H, 8.27.

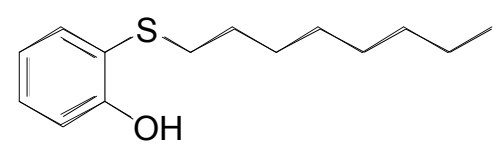

2-Octylsulfanylphenol (Table 1, entry 29). ${ }^{13} \mathrm{~A}$ solution of $\mathrm{Pd}(\mathrm{OAc})_{2}(4.4 \mathrm{mg})$ and CyPF- $t$ $\mathrm{Bu}(11 \mathrm{mg})$ in DME $(1 \mathrm{~mL})$ was used as catalyst. $97 \%$ yield. Yellow liquid. ${ }^{1} \mathrm{H} \mathrm{NMR}\left(\mathrm{CDCl}_{3}\right)$ : $\delta 7.38(\mathrm{dd}, J=7.6 \mathrm{~Hz}$ and $1.5 \mathrm{~Hz}, 1 \mathrm{H}), 7.17(\mathrm{dt}, J=7.6 \mathrm{~Hz}$ and $1.5 \mathrm{~Hz}, 1 \mathrm{H}), 6.90(\mathrm{~d}, J=8.1 \mathrm{~Hz}$, $1 \mathrm{H}), 6.80-6.76(\mathrm{~m}, 1 \mathrm{H}), 6.70(\mathrm{bs}, 1 \mathrm{H}), 2.60(\mathrm{t}, J=7.5 \mathrm{~Hz}, 2 \mathrm{H}), 1.50-1.43(\mathrm{~m}, 2 \mathrm{H}), 1.31-1.17(\mathrm{~m}$, $10 \mathrm{H}), 0.79(\mathrm{t}, J=6.8 \mathrm{~Hz}, 3 \mathrm{H}) .{ }^{13} \mathrm{C} \mathrm{NMR}\left(\mathrm{CDCl}_{3}\right): \delta 156.8,135.8,130.8,120.6,119.1,114.6$, $36.7,31.7,29.5,29.04,28.98,28.5,22.5,14.0$.

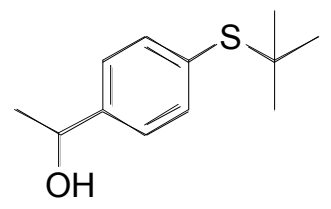

4-(1-Hydroxyethyl)phenyl 2-methyl-2-propyl sulfide (Table 1, entry 30). $500 \mu \mathrm{L}$ of stock solution $\mathbf{A}$ and $\mathrm{NaO} t \mathrm{Bu}(98 \mathrm{mg}, 1.02 \mathrm{mmol})$ were used. 93\% yield. Colorless oil. ${ }^{1} \mathrm{H}$ NMR $\left(\mathrm{CDCl}_{3}\right): \delta 7.50(\mathrm{~d}, J=8.0 \mathrm{~Hz}, 2 \mathrm{H}), 7.33(\mathrm{~d}, J=8.0 \mathrm{~Hz}, 2 \mathrm{H}), 4.91(\mathrm{q}, J=6.6 \mathrm{~Hz}, 1 \mathrm{H}), 1.95(\mathrm{bs}$, 
$1 \mathrm{H}), 1.50(\mathrm{~d}, J=6.6 \mathrm{~Hz}, 3 \mathrm{H}), 1.28(\mathrm{~s}, 9 \mathrm{H}) .{ }^{13} \mathrm{C} \mathrm{NMR}\left(\mathrm{CDCl}_{3}\right): \delta 146.3,137.5(2 \mathrm{C}), 131.5,125.4$ (2C), 70.0, 45.8, 30.9 (3C), 25.1. Elem. Anal. Calcd for $\mathrm{C}_{12} \mathrm{H}_{18} \mathrm{OS}$ : C, 68.52; H, 8.63. Found: C, 68.49; H, 8.63.

General procedure for the palladium-catalyzed coupling of aryl bromides and iodides with thiols: The appropriate quantity of stock solution $\mathbf{A}$ or $\mathbf{C}$ was added to a $4 \mathrm{~mL}$ vial containing the aryl halide $(1.00 \mathrm{mmol})$ and sodium tert-butoxide $(106 \mathrm{mg}, 1.10 \mathrm{mmol})$ in $1.5 \mathrm{~mL}$ of DME. The thiol $(1.00 \mathrm{mmol})$ was then added, and the vial sealed with a cap containing a PTFE septum. The mixture was heated at the temperature indicated in table 2 until the aryl halide was consumed, as determined by GC. Silica gel (0.5 g) was added, and the solvents were evaporated under reduced pressure. The crude residue was purified by column chromatography on silica gel using hexane as eluent. Aryl sulfides were isolated in the yields reported in Table 2.

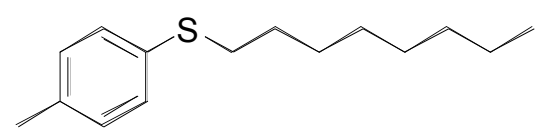

4-Methylphenyl octyl sulfide (Table 2 , entry 1). ${ }^{1} 100 \mu \mathrm{L}$ of stock solution $\mathbf{C}$ were used, and the mixture heated at $110{ }^{\circ} \mathrm{C} .99 \%$ yield. Colorless liquid. ${ }^{1} \mathrm{H}$ NMR $\left(\mathrm{CDCl}_{3}\right): \delta 7.10(\mathrm{~d}, J=$ $8.1 \mathrm{~Hz}, 2 \mathrm{H}), 6.95(\mathrm{~d}, J=8.1 \mathrm{~Hz}, 2 \mathrm{H}), 2.73(\mathrm{t}, J=7.4 \mathrm{~Hz}, 2 \mathrm{H}), 2.17$ (s, 3H), 1.48 (quint, $J=7.4$ $\mathrm{Hz}, 2 \mathrm{H}), 1.26$ (quint, $J=7.4 \mathrm{~Hz}, 2 \mathrm{H}), 1.17-1.08(\mathrm{~m}, 8 \mathrm{H}), 0.74(\mathrm{t}, J=6.9 \mathrm{~Hz}, 3 \mathrm{H}) .{ }^{13} \mathrm{C} \mathrm{NMR}$ $\left(\mathrm{CDCl}_{3}\right): \delta 135.7,133.1,129.7$ (2C), 129.5 (2C), 34.3, 31.7, 29.2, 29.10, 29.06, 28.7, 22.6, 20.9, 14.0.

4-Methylphenyl octyl sulfide (Table 2, entry 2). $50 \mu \mathrm{L}$ of stock solution A were used, and the mixture heated at $90{ }^{\circ} \mathrm{C} .96 \%$ yield.

4-Methylphenyl octyl sulfide (Table 2, entry 3). $1000 \mu \mathrm{L}$ of stock solution A were used, and the mixture heated at $50{ }^{\circ} \mathrm{C} .97 \%$ yield. 
4-Methylphenyl phenyl sulfide (Table 2, entry 4). ${ }^{2} 10 \mu \mathrm{L}$ of stock solution A were used, and the mixture heated at $110^{\circ} \mathrm{C} .98 \%$ yield.

4-Methylphenyl phenyl sulfide (Table 2, entry 5). $100 \mu \mathrm{L}$ of stock solution A were used, and the mixture heated at $90{ }^{\circ} \mathrm{C} .99 \%$ yield.

4-Methylphenyl phenyl sulfide (Table 2, entry 6). $1000 \mu \mathrm{L}$ of stock solution A were used, and the mixture heated at $50{ }^{\circ} \mathrm{C} .91 \%$ yield.

4-Methylphenyl octyl sulfide (Table 2, entry 7). ${ }^{1} 100 \mu \mathrm{L}$ of stock solution $\mathbf{C}$ were used, and the mixture heated at $110{ }^{\circ} \mathrm{C} .84 \%$ yield (90\% conversion).

4-Methylphenyl octyl sulfide (Table 2, entry 8). $10 \mu \mathrm{L}$ of stock solution A were used, and the mixture heated at $90{ }^{\circ} \mathrm{C} .97 \%$ yield.

4-Methylphenyl octyl sulfide (Table 2, entry 9). $500 \mu \mathrm{L}$ of stock solution A were used, and the mixture stirred at room temperature. $96 \%$ yield.

4-Methylphenyl phenyl sulfide (Table 2 , entry 10). ${ }^{2} 10 \mu \mathrm{L}$ of stock solution A were used, and the mixture heated at $110{ }^{\circ} \mathrm{C} .82 \%$ yield (90\% conversion).

4-Methylphenyl phenyl sulfide (Table 2, entry 11). $10 \mu \mathrm{L}$ of stock solution A were used, and the mixture heated at $90{ }^{\circ} \mathrm{C} .95 \%$ yield.

4-Methylphenyl phenyl sulfide (Table 2, entry 12). $500 \mu \mathrm{L}$ of stock solution A were used, and the mixture stirred at room temperature. $97 \%$ yield.

General procedure for the palladium-catalyzed coupling of aryl sulfonates with thiols: The appropriate quantity of catalyst was added to a $4 \mathrm{~mL}$ vial containing the aryl sulfonate (1.00 $\mathrm{mmol})$ and potassium carbonate $(152 \mathrm{mg}, 1.10 \mathrm{mmol})$ or sodium carbonate $(117 \mathrm{mg}, 1.10 \mathrm{mmol})$ in $1.5 \mathrm{~mL}$ of DME. The thiol $(1.00 \mathrm{mmol})$ was then added, and the vial sealed with a cap containing a PTFE septum. The mixture was heated at $110{ }^{\circ} \mathrm{C}$ until the aryl sulfonate was 
consumed, as determined by GC. Silica gel $(0.5 \mathrm{~g})$ was added, and the solvents were evaporated under reduced pressure. The crude residue was purified by column chromatography on silica gel using hexane as eluent. Aryl sulfides were isolated in the yields reported in Table 2.

Octyl phenyl sulfide (Table 2, entry 13). ${ }^{3} 250 \mu \mathrm{L}$ of stock solution A were used. 95\% yield.

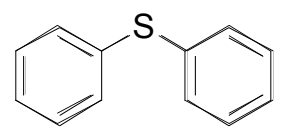

Diphenyl sulfide (Table 2, entry 14). ${ }^{2}$ A solution of $\mathrm{Pd}(\mathrm{OAc})_{2}(4.4 \mathrm{mg})$ and CyPF- $t$-Bu (11 $\mathrm{mg})$ in DME $(1 \mathrm{~mL})$ was used as catalyst. $86 \%$ yield. Colorless liquid. ${ }^{1} \mathrm{H}$ NMR $\left(\mathrm{CDCl}_{3}\right): \delta 7.26-$ $7.24(\mathrm{~m}, 4 \mathrm{H}), 7.21-7.18(\mathrm{~m}, 4 \mathrm{H}), 7.16-7.13(\mathrm{~m}, 2 \mathrm{H}) .{ }^{13} \mathrm{C} \mathrm{NMR}\left(\mathrm{CDCl}_{3}\right): \delta 135.7(2 \mathrm{C}), 130.9$ (4C), $129.1(4 \mathrm{C}), 126.9(2 \mathrm{C})$.

Octyl phenyl sulfide (Table 2, entry 15). A solution of $\mathrm{Pd}(\mathrm{OAc})_{2}(4.4 \mathrm{mg})$ and $\mathrm{CyPF}-t$-Bu $(11 \mathrm{mg})$ in DME $(1 \mathrm{~mL})$ was used as catalyst. $79 \%$ yield ( $90 \%$ conversion).

Synthesis of triisopropylphenylthiosilane: $100 \mu \mathrm{L}$ of stock solution $\mathbf{A}$ was added to a $4 \mathrm{~mL}$ vial containing bromobenzene $(157 \mathrm{mg}, 1.00 \mathrm{mmol})$ and LiHMDS (lithium bis(trimethylsilyl)amide) (184 mg, $1.10 \mathrm{mmol})$ in $1.5 \mathrm{~mL}$ of toluene. Triisopropylsilanethiol (190 $\mathrm{mg}, 1.00 \mathrm{mmol}$ ) was then added, and the vial sealed with a cap containing a PTFE septum. The mixture was heated at $90{ }^{\circ} \mathrm{C}$ until bromobenzene was consumed $(2 \mathrm{~h})$, as determined by GC. Silica gel $(0.5 \mathrm{~g})$ was added, and solvents were evaporated under reduced pressure. The crude residue was purified by column chromatography on silica gel using hexane as eluent giving 242 mg (91\% yield) of colorless triisopropylphenylthiosilane. 


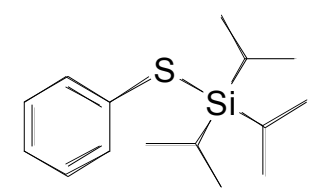

Phenyltriisopropylsilane. ${ }^{14} 91 \%$ yield. Colorless liquid. ${ }^{1} \mathrm{H}$ NMR $\left(\mathrm{CDCl}_{3}\right): \delta$ 7.41-7.39 (m, 2H), 7.11-7.09 (m, 3H), 1.19-1.11 (m, 3H), $0.99(\mathrm{~d}, J=7.3 \mathrm{~Hz}, 18 \mathrm{H}) .{ }^{13} \mathrm{C}$ NMR $\left(\mathrm{CDCl}_{3}\right)$ : $\delta 135.4(2 \mathrm{C}), 131.4,128.5(2 \mathrm{C}), 126.6,18.3(6 \mathrm{C}), 13.0(3 \mathrm{C})$.

Synthesis of 4-Methylphenyl phenyl sulfide from phenyltriisopropylsilane: $\mathrm{Pd}(\mathrm{OAc})_{2}$ $(1.1 \mathrm{mg})$ and CyPF-t-Bu $(2.75 \mathrm{mg})$ were diluted in DME $(0.5 \mathrm{~mL})$ and added to a $4 \mathrm{~mL}$ vial containing $p$-bromotoluene ( $86 \mathrm{mg}, 0.50 \mathrm{mmol})$ and $\mathrm{CsF}$ (228 mg, 3 equiv., $1.50 \mathrm{mmol}$ ) in $1 \mathrm{~mL}$ of toluene. Triisopropylphenylthiosilane $(133 \mathrm{mg}, 0.50 \mathrm{mmol})$ was then added, and the vial sealed with a cap containing a PTFE septum. The mixture was heated at $70{ }^{\circ} \mathrm{C}$ until $p$ bromotoluene was consumed, as determined by GC. Silica gel $(0.5 \mathrm{~g})$ was added, and the solvents were evaporated under reduced pressure. The crude residue was purified by column chromatography on silica gel using hexane as eluent giving $88 \mathrm{mg}$ ( $88 \%$ yield) of 4 methylphenyl phenyl sulfide.

(1) Yoon, N. M.; Choi, J.; Ahn, J. H. J. Org. Chem. 1994, 59, 3490-3493.

(2) Deng, W.; Zou, Y.; Wang, Y.-F.; Liu, L.; Guo, Q.-X. Synlett 2004, 1254-1258.

(3) Barbero, M.; Degani, I.; Diulgheroff, N.; Dughera, S.; Fochi, R.; Migliaccio, M. J. Org. Chem. 2000, 65, 5600-5608.

(4) Herradura, P. S.; Pendola, K. A.; Guy, R. K. Org. Lett. 2000, 2, 2019-2022.

(5) Bates, C. G.; Gujadhur, R. K.; Venkataraman, D. Org. Lett. 2002, 4, 2803-2806.

(6) Katritzky, A. R.; Lue, P. J. Org. Chem. 1990, 55, 74-78.

(7) Robert, I.; Higgins, W.; Garrett, R. J. Org. Chem. 1962, 27, 2168-2170.

(8) Olah, G. A.; Vankar, Y. D.; Arvanaghi, M. Synthesis 1979, 984-985.

(9) Murata, M.; Buchwald, S. L. Tetrahedron 2004, 60, 7397-7403.

(10) Bunnett, J. F.; Rauhut, M. M. J. Org. Chem. 1956, 21, 934-938.

(11) Kwong, F. Y.; Buchwald, S. L. Org. Lett. 2002, 4, 3517-3520.

(12) Rae, I. D. Can. J. Chem. 1965, 43, 2614-2616.

(13) Kalgutkar, A. S.; Kozak, K. R.; Crews, B. C.; Hochgesang, G. P., Jr.; Marnett, L. J. J. Med. Chem. 1998, 41, 4800-4818.

(14) Rane, A. M.; Miranda, E. I.; Soderquist, J. A. Tetrahedron Lett. 1994, 35, 3225-3226. 